\title{
$\left[{ }^{11} \mathrm{C}\right]$ acetate PET/CT Visualizes Skeletal Muscle Exercise Participation, Impaired Function, and Recovery after Hip Arthroplasty; First Results
}

\author{
Franz Buchegger, ${ }^{1}$ Osman Ratib, ${ }^{1}$ Jean-Pierre Willi, ${ }^{1}$ Charles Steiner, ${ }^{1}$ Yann Seimbille, ${ }^{1}$ \\ Habib Zaidi, ${ }^{1}$ Véronique Graf, ${ }^{2}$ Robin Peter, ${ }^{2}$ Maximilien Jung ${ }^{2}$ \\ ${ }^{1}$ Service of Nuclear Medicine, University Hospital of Geneva, Rue Gabrielle Perret-Gentil 4, CH 1211, Geneva 14, Switzerland \\ ${ }^{2}$ Service of Orthopedic Surgery, University Hospital of Geneva, Geneva, Switzerland
}

\begin{abstract}
Purpose: Based on skeletal muscle acetate physiology we aimed studying muscle function after hip arthroplasty with $\left[{ }^{11} \mathrm{C}\right]$ acetate PET.

Procedures: Two male patients were investigated 3 and 12 weeks after hip arthroplasty with muscle $\left[{ }^{11} \mathrm{C}\right]$ acetate $\mathrm{PET} / \mathrm{CT}$ performed at rest and exercise. Median muscle SUV $\mathrm{V}_{\text {mean }}$ were calculated on three non-consecutive transverse PET slices.

Results: The four exercise PET/CT showed, compared with rest, consistent increase in $\left[{ }^{11} \mathrm{C}\right]$ acetate uptake in active muscles contralateral to surgery. On the arthroplasty side most muscles showed symmetric activity increase under exercise both at 3 and 12 weeks after surgery, but four muscles showed only minor activity increase at 3 weeks. At 3 months, functional recovery of the latter four muscles was observed.

Conclusion: Consistent increase in $\left[{ }^{11} \mathrm{C}\right]$ acetate uptake in healthy muscles under exercise compared with rest was observed by PET/CT. Transiently impaired muscle function 3 weeks after surgery recovered at 3 months. These first observations merit further investigation.
\end{abstract}

Key words: $\left[{ }^{11} \mathrm{C}\right]$ acetate, PET/CT, Skeletal muscle exercise PET, Hip arthroplasty, Muscle injury

\section{Introduction}

A cetate consumption in resting human contributes to less than $10 \%$ of global $\mathrm{CO}_{2}$ production when measured with $\left[{ }^{14} \mathrm{C}\right]$ acetate [1]. In resting, human acetate is mainly metabolized in the liver [2, 3].

In 2002, van Hall et al. showed that $\left[{ }^{13} \mathrm{C}\right]$ acetate turnover in active skeletal muscle was greatly increased compared with rest and represented, during exercise, a major part of whole body acetate turnover [4]. These authors studied the kinetics of increase and decrease of $\left[{ }^{13} \mathrm{C}\right]$ acetate in peripheral

Research article demonstrating $\left[{ }^{11} \mathrm{C}\right]$ acetate PET/CT imaging of skeletal muscle exercise participation

Correspondence to: Franz Buchegger; e-mail: Franz.Buchegger@hcuge.ch blood under constant infusion of acetate, measurement intervals being spaced by $30 \mathrm{~min}$ after initiation and later stopping of exercise [4]. Higashi et al., furthermore, while studying the kinetics of $\left[{ }^{11} \mathrm{C}\right]$ acetate uptake in lung cancer, showed that heart uptake peaked a few minutes after injection, remained maximal over about $6 \mathrm{~min}$ before decreasing again during the next $20 \mathrm{~min}$ [5].

We hypothesized that skeletal muscle $\left[{ }^{11} \mathrm{C}\right]$ acetate uptake might be visualized by positron emission tomography (PET) in analogy to heart muscle activity. We thus designed an exercise related with the one shown in the report of Van Hall et al. [4] but also respecting particular constraints of our patients and the kinetics of $\left[{ }^{11} \mathrm{C}\right]$ acetate uptake in the heart described by Higashi et al. [5]. For this purpose, we used an exercise force development adequate for patients in the postsurgical situation after hip arthroplasty and started PET imaging shortly after exercise in order to remain within the 
window of maximal $\left[{ }^{11} \mathrm{C}\right]$ acetate uptake demonstrated for heart muscle [5].

This article gives the first results with $\left[{ }^{11} \mathrm{C}\right]$ acetate muscle function PET performed in two patients after hip arthroplasty. Both patients were studied with rest and exercise PET/computed tomography (CT) once after 3 weeks and again after reeducation at 3 months.

\section{Patients and Methods}

Patients gave their written informed consent to the study protocol that had been approved by the Medical Ethical Committee, Geneva University Hospital, by Swissmedic and by the Federal Office of Public Health, Section of Radioprotection.

Male patients were eligible after first, single-sided hip arthroplasty. This allowed the analysis of muscle exercise participation on the surgical side compared with the healthy body side. Both patients participated in a reeducation program of 3 months.

\section{Hip Arthroplasty}

Patient 1 had a left-side hip arthroplasty through the lateral transglutaeal (Hardinge) approach (Table 1). Patient 2 had a right-side hip arthroplasty through the posterior ("Kocher-Langenbeck") approach. A short shaft prothesis was implanted in the latter patient and standard uptake values (SUV) measurements could be performed at the glutaeus and obturator muscle levels outside of the CT artifact.

\section{$\left[{ }^{11}\right.$ C]acetate}

1- $\left[{ }^{11} \mathrm{C}\right]$ acetate $\left(\left[{ }^{11} \mathrm{C}\right]\right.$ acetate) of clinical grade (GCP, GMP) was prepared at the cyclotron unit of Geneva University Hospital from $\left[{ }^{11} \mathrm{C}\right]$ carbon dioxide, produced itself on an IBA 18/9 cyclotron, as described [6].

\section{PET/CT}

Studies were performed on the LSO-based PET/CT scanner Biograph 64 (Siemens Medical Solutions, Erlangen, Germany). Patients were requested to restrain from heavy muscle exercise for $24 \mathrm{~h}$ prior to PET, a light morning meal $6 \mathrm{~h}$ before $\left[{ }^{11} \mathrm{C}\right]$ acetate PET was being permitted. Patients were positioned on the camera bed and remained relaxed for $10 \mathrm{~min}$. For their comfort, an articulated knee position was accepted using a knee support of about 5-7 cm.

Table 1. Patient characteristics and surgical procedure

\begin{tabular}{|c|c|c|}
\hline & Patient I & Patient II \\
\hline Age (years) & 59 & 58 \\
\hline Arthroplasty & Left hip & Right hip \\
\hline Surgical approach & $\begin{array}{l}\text { Lateral (transglutaeal; } \\
\text { Hardinge) }\end{array}$ & $\begin{array}{l}\text { Posterior } \\
\quad \text { (Kocher-Langenbeck) }\end{array}$ \\
\hline Femoral device & Long shaft & Short shaft \\
\hline
\end{tabular}

For the rest PET/CT, $200 \mathrm{MBq}(5.4 \mathrm{mCi})\left[{ }^{11} \mathrm{C}\right]$ acetate was injected i.v. A low-dose CT $(120 \mathrm{kVp}, 60 \mathrm{mAs}, 16 \times 1.5$ collimation, pitch of 0.8 and $0.5 \mathrm{~s}$ /rotation) was registered over two bed positions between the iliac crest and the upper leg. PET scanning, 4 min per bed position, was then performed, starting $5 \mathrm{~min}$ after tracer injection.

For exercise PET, a new activity of 800 or $600 \mathrm{MBq}\left[{ }^{11} \mathrm{C}\right]$ acetate was then injected i.v. in the first and second patient, respectively. Immediately after injection patients performed 20 isometric bilateral abductor exercises of $10 \mathrm{~s}$ followed by a relaxation time of $3 \mathrm{~s}$ each with sphygmomanometer-controlled pressure limited to $100 \mathrm{mmHg}$ against the ankles using a physical constraint. A second low-dose CT was again registered followed directly by PET scanning of 3 min per bed position.

Following Fourier rebinning and model-based scatter correction, PET images were reconstructed using two-dimensional iterative normalized attenuation-weighted ordered subsets expectation maximization [7] and CT-based attenuation correction.

\section{PET/CT Interpretation}

PET/CT was interpreted by two experienced nuclear medicine physicians. Mean standard uptake values $\left(\mathrm{SUV}_{\text {mean }}\right)$ over three nonconsecutive slices [8] were calculated within regions of interest (ROIs) drawn over individual muscles as defined by $\mathrm{CT}$, using standard formula [9]. ROIs were drawn outside the prothesis CT artifact over the entire muscle transverse sections.

\section{Statistical Evaluation}

Results of SUV measurements in different groups of muscles as presented in Fig. 3 were compared using the Student-Newman-Keuls multigroup comparison at different significance levels (Unistat 5.6, statistical package for Windows, UNISTAT Ltd, London). $p<0.05$ was considered significant, $p<0.001$ as highly significant.

\section{Results}

\section{Visual Interpretation of PET/CT}

At 3 weeks after surgery, the four exercise PET/CT showed, on the healthy body side in both patients, a strong increase of $\left[{ }^{11} \mathrm{C}\right]$ acetate uptake in the abductor muscles as compared with rest, notably the small, median, and the external fibers of the large glutaeus muscle (Fig. 1, patient 2). A similar increase in uptake under exercise was also observed in the iliac and psoas (Fig. 1a) and internal obturator muscles (Fig. 1b) above and below the implant artifact, respectively.

On the arthroplasty side, most muscles showed in symmetry a similar exercise uptake increase. Rest activity was slightly higher on the surgical side compared with the contralateral side. Clearly, three muscles on the arthroplasty side of the patient having undergone the posterior "Kocher Langenbeck" arthroplasty approach (Fig. 1), namely the iliac and psoas muscles (Fig. 1a) and the internal obturator 
a
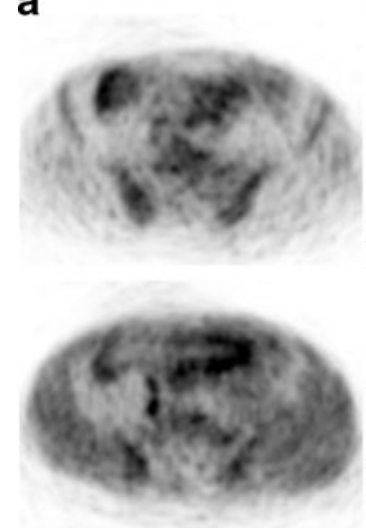

b
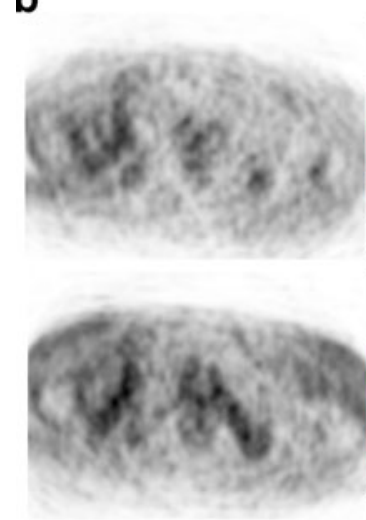
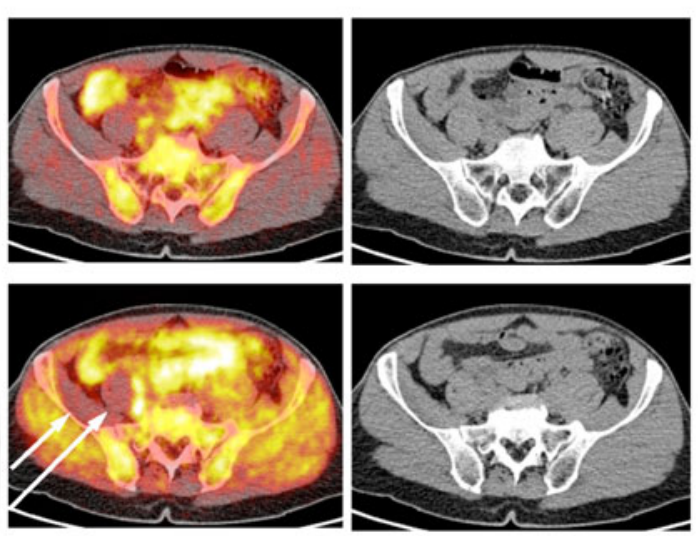

Exercise
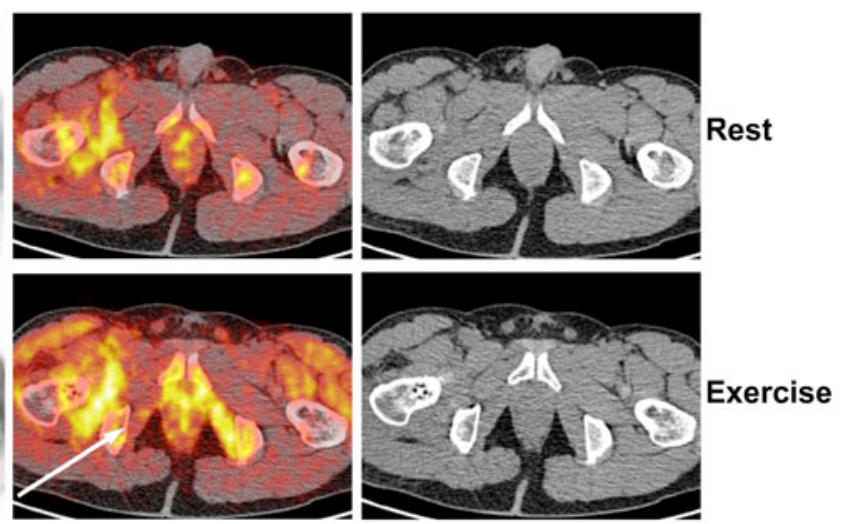

Fig. 1. $\left[{ }^{11} \mathrm{C}\right]$ acetate $\mathrm{PET} / \mathrm{CT} 3$ weeks after right hip arthroplasty through the posterior "Kocher Langerhans" approach. Imaging at the level of the iliac and psoas (a) and obturator muscles (b) is shown with the upper row showing PET at rest and the lower row PET after exercise. Each line shows, from left to right, PET alone, PET/CT superposition and the low-dose CT alone. Note that the three glutaeal muscles (a) (the small, the median, and the large glutaeus muscle with the external fibers) responded with strong increase in $\left[{ }^{11} \mathrm{C}\right]$ acetate uptake during the abductor exercise (lower row) compared with rest (upper row). A strong increase in $\left[{ }^{11} \mathrm{C}\right]$ acetate uptake under exercise was also observed on the healthy body side in the iliac and psoas (a) and internal obturator (b) muscles, while on the surgical side these latter muscles (arrows) show similar activity under rest and exercise at 3 weeks. The postsurgical $\left[{ }^{11} \mathrm{C}\right]$ acetate PET hyperactivity extending into the external obturator muscle on the right side, directly related to the surgical approach, remained stable at rest, and exercise.

muscle (Fig. 1b) did not show any relevant increase in uptake under exercise, in contrast to the increase in these same muscles on the contralateral healthy body side.

In the other patient, having undergone a lateral "Hardinge" transgluteal arthroplasty approach, the small glutaeus muscle and the external fibers of the large glutaeus muscle showed a similar increase in $\left[{ }^{11} \mathrm{C}\right]$ acetate uptake under exercise as the contralateral muscles. However the median glutaeus muscle showed only a marginal increase in uptake under exercise at 3 weeks (result not shown) compared with rest. The rest activity of the median gluteaus muscle at 3 weeks was markedly above the rest activity in the adjacent and contralateral muscles.

These same muscle groups were again reevaluated using an identical study protocol 3 months after surgery, both patients having completed their reeducation program. At the second PET evaluation, a similar increase in $\left[{ }^{11} \mathrm{C}\right]$ acetate uptake as of 3 weeks was again observed in all muscles that participated previously in the exercise (Fig. 2). Furthermore, the four previously inactive muscles on the surgery side had clearly regained activity by showing an increase in $\left[{ }^{11} \mathrm{C}\right]$ acetate uptake under exercise very similar to all other active muscles.

\section{Additional Observations}

In both patients, a marked hyperactivity related with the surgical intervention was observed at 3 weeks and was only partially resolved at 3 months. PET showed also a postsurgical collection in the first patient adjacent to the large gluteus muscle at the level of the greater trochanter. 

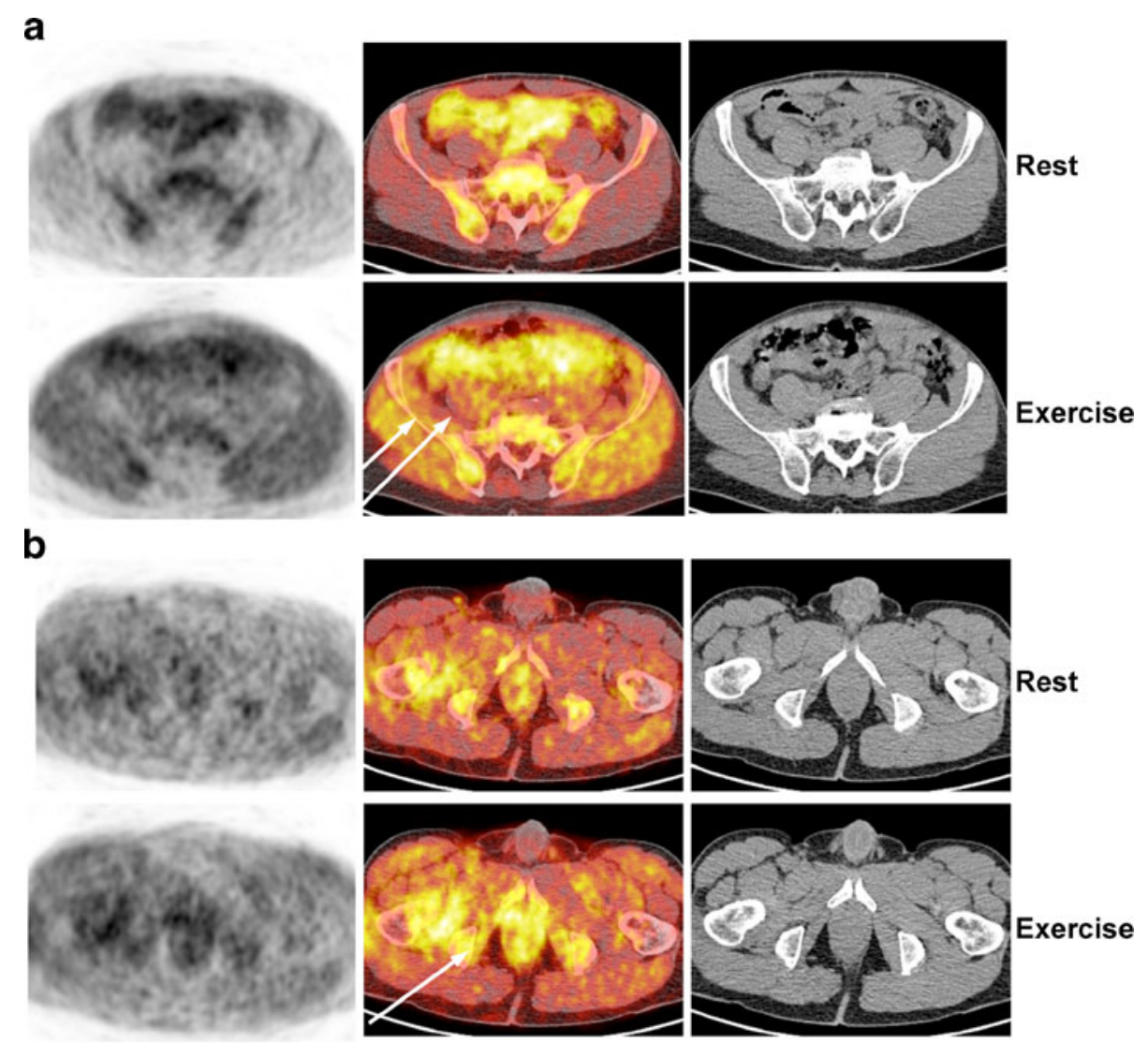

Fig. 2. PET/CT imaging at 3 months post surgery of the same patient as shown in Fig. 1. Figure arrangement is as described under Fig. 1. Note that imaging on the level of the iliac and psoas muscles (a) and internal obturator (b) shows the almost complete recovery of the previously stunned iliac and psoas and internal obturator muscles (arrows) on the surgery side. Exercise (lower row) increase in $\left[{ }^{11} \mathrm{C}\right]$ acetate uptake on the surgery side is now similar to that on the healthy body side also for these muscles. The three glutaeal muscles (a) responded again well at 3 months to the abductor exercise. Note that the postsurgical hyperactivity, reaching into the external obturator muscle, is still well visualized at 3 months post surgery but was no obstacle to good participation in the exercise of the nearby internal obturator muscle.

The observation of a global prostate hyperactivity observed in one of the two patients was also reported to his physician.

\section{SUV $V_{\text {mean }}$ Measurements in CT Delimitated Muscle Groups}

In the four exercise PET/CTs, the average $\mathrm{SUV}_{\text {mean }}$ of $\left[{ }^{11} \mathrm{C}\right]$ acetate activity significantly increased above that of rest in the healthy abductor muscles and in the ilio-psoas and the internal obturator muscles contralateral to surgery. At 3 weeks post surgery, the increase was 2.9 -fold above rest activity $(p<0.001)$ while at 3 months the increase was 2.1 fold above rest $(p<0.001)$. In these muscles, the median $\mathrm{SUV}_{\text {mean }}$ at rest was $0.79 \pm 0.15$ and $0.76 \pm 0.22$ at 3 weeks and 3 months, respectively, and increased after exercise to $2.33 \pm 0.51$ (Fig. 3) and 1.56 \pm 0.34 , respectively (Fig. 3, group A).

Muscles apparently not harmed on the surgical side showed a similar increase in uptake under exercise from a basal $0.91 \pm 0.21-1.93 \pm 0.41$ at 3 weeks (exercise activity 2.1-fold above rest) while at 3 months increase in uptake was 1.7 -fold above rest activity rising from basal $0.76 \pm$ $0.26-1.32 \pm 0.24(p<0.001)$ after exercise.

However, at 3 weeks post surgery the four impaired muscles showed hardly any measurable increase in $\mathrm{SUV}_{\text {mean }}$ with a mean of $1.05 \pm 0.33$ at rest and $1.19 \pm 0.17$ under exercise (Fig. 3, $p>0.5$ ). At 3 months, increase in activity of muscles under exercise $\left(\mathrm{SUV}_{\text {mean }} 1.52 \pm 0.09\right)$ was restored being 1.7-fold above the rest $\operatorname{SUV}_{\text {mean }}(0.88 \pm 0.18),(p<$ $0.001)$. This latter increase in activity of previously injured muscles was notably identical to the measured increase of non-injured muscles on the surgery side at 3 months.

For comparison, the visually inactive obturator external muscle was measured both at initial PET and after 3 months and showed on all examinations a very similar activity at rest and after exercise with only a marginal increase under exercise (group D, Fig. 3, $p>0.8$ ).

\section{Discussion}

Imaging with $\left[{ }^{18} \mathrm{~F}\right] \mathrm{FDG}$ PET showed the functioning of skeletal muscles by performing specific muscle exercises after injection of patients evaluated for suspected neoplasic 


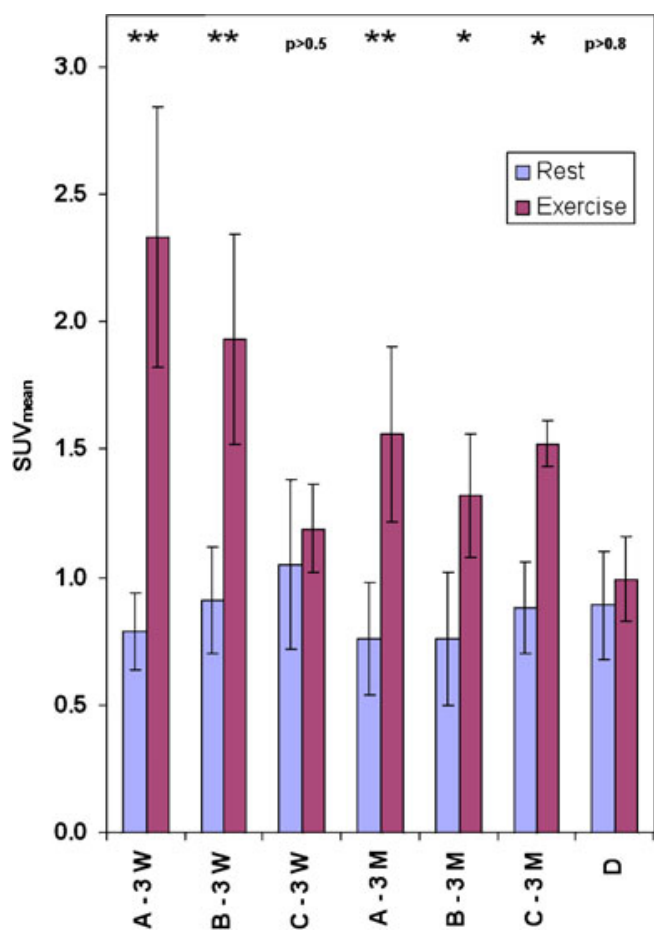

Fig. 3. Histogram showing median activity at rest and exercise \pm 1 standard deviation (SD) of $S U V_{\text {mean }}$ for different groups of muscles evaluated at 3 weeks $(3 W$ ) and 3 months (3 $M$ ) post surgery, respectively, for the two patients. Responding muscles on the healthy body side (group A) comprised the large (two), median (two), and small (two) glutaeus, the iliac (two) and psoas (two), and internal obturator (one; $N=11$ ). Active muscles on the surgery side (group B) comprised the large (two), median (one), and small glutaeus (two), the iliac (one) and psoas (one) muscles $(N=7)$. Muscles stunned in the post arthroplasty situation at 3 weeks and recovered at 3 months (group $\mathrm{C}$ ) comprised the median glutaeus (one), the iliac (one), and psoas (one) and the internal obturator (one) muscles $(N=4)$. As an inactive muscle $(D$; visually not participating in the exercise), the external obturator muscle was measured on both body sides (on the surgery side outside the stable postsurgical hyperactivity) in patient 2 (Fig. 1 and 2) at 3 weeks and 3 months $(N=4$, paired measurements). The symbols one asterisk and two asterisks indicate significant and highly significant differences, respectively, between rest and exercise as calculated with the Student-Newman-Keuls multigroup statistical comparison.

disease [10]. It was shown that $\left[{ }^{18} \mathrm{~F}\right] \mathrm{FDG}$ uptake increased specifically in exercising muscles compared with resting muscle groups. Aiming to reduce radiation exposure in muscle function PET and giving preference, notably in the postsurgical situation, to a double PET study at rest and after exercise, we hypothesized that muscle function PET could possibly be performed with $\left[{ }^{11} \mathrm{C}\right]$ acetate.

Our first results clearly show a significant increase in $\left[{ }^{11} \mathrm{C}\right]$ acetate uptake under exercise compared with rest in healthy abductor muscles. Other muscles without abductor function were also activated on both body sides. This latter observation may be related to the previous observation that $\left[{ }^{13} \mathrm{C}\right]$ acetate extraction from blood was also increased in the resting leg which should not actively participate in the exercise [4]. Furthermore, allowing a minor knee articulation position for our patients aimed at permitting complete muscle relaxation, the intended abductor exercise also required some corrective exercise of the inner rotation muscles in order to counteract an external rotation tendency under the exercise pressure to both ankles.

At 3 months, all active muscles on the healthy body side were also symmetrically active on the surgery side, indicating the absence of any incidental single-sided muscle acetate uptake not related to the symmetric exercise. However, at 3 weeks post surgery, four muscles directly related to the surgical procedure showed absence of exercise participation as visualized by absence of significant increase in $\left[{ }^{11} \mathrm{C}\right]$ acetate uptake. Whether the absence of muscle function observed at 3 weeks post surgery was the product of a direct muscle or an activation failure, such as seen in painful osteoarthritic knees, remains an open question. The regained exercise participation of these four muscles at 3 months post surgery clearly shows that muscle function impairment was temporary, unrelated to any definitive nerve damage. A nervous lesion seems unlikely since the reinervation time would be longer than the 3 months, after which time, recovered muscle function could be demonstrated.

$\left[{ }^{11} \mathrm{C}\right]$ acetate PET of heart muscle has been described by different authors [11-13]. $\left[{ }^{11} \mathrm{C}\right]$ acetate undergoes a high first-pass extraction in the heart muscle and enters a first and second tricarboxylic acid (TCA) cycle with production of different intermediate metabolites before being converted into $\left[{ }^{11} \mathrm{C}\right] \mathrm{CO}_{2}$ in the second TCA cycle [14]. Residence time of $\left[{ }^{11} \mathrm{C}\right]$ acetate in the heart is short $[5,14]$ due to metabolism of $\left[{ }^{11} \mathrm{C}\right]$ acetate into $\left[{ }^{11} \mathrm{C}\right] \mathrm{CO}_{2}$. The kinetics of $\left[{ }^{11} \mathrm{C}\right]$ acetate clearance from heart muscle, as measured by dynamic PET, has been proposed as a means to estimate myocardial oxygen consumption [15-17] and has also been shown to be related to ventricular efficiency [18].

Acetate metabolism of skeletal muscle is less well known. Non-oxidative reactions in the TCA cycle also produce different metabolites and oxidative production of $\left[{ }^{13} \mathrm{C}\right] \mathrm{CO}_{2}$ has been described to be relatively slow in resting muscle [4]. With exercise, however, oxidative production of $\mathrm{CO}_{2}$ from $1-\left[{ }^{14} \mathrm{C}\right]$ acetate in skeletal muscle would be significantly more rapid than at rest. In exercising volunteers, $\left[{ }^{14} \mathrm{C}\right] \mathrm{CO}_{2}$ in breath decreased by $50 \% 20 \mathrm{~min}$ after infusion stop of $1-\left[{ }^{14} \mathrm{C}\right]$ acetate [19], suggestive of a relatively short-term metabolic consumption of acetate.

Our PET imaging time from 5 to $13 \mathrm{~min}$ and 5 to $11 \mathrm{~min}$ postinjection in rest and exercise, respectively, was designed to match the frame of maximal $\left[{ }^{11} \mathrm{C}\right]$ acetate uptake in heart muscle. Imaging time was thus clearly beyond perfusion measurement time adopted in heart [20]. We thus believe that we observed a real increase in $\left[{ }^{11} \mathrm{C}\right]$ acetate muscle uptake under exercise. However, in analogy to heart muscle measurements, dynamic imaging of uptake and particularly elimination phase of $\left[{ }^{11} \mathrm{C}\right]$ acetate in resting and exercising muscle may be required to prove an increase of $\left[{ }^{11} \mathrm{C}\right]$ acetate consumption rate by exercising muscle. 
Different aspects of $\left[{ }^{11} \mathrm{C}\right]$ acetate muscle metabolism remained unclear in the preparation of this pilot study. Thus, under different study protocols, $\left[{ }^{13} \mathrm{C}\right]$ acetate had been infused in volunteers at micromolar concentration and it was shown that acetate consumption rose over longer periods of time [2-4] and did not reach its peak after $3 \mathrm{~h}$ [3], suggesting the need for an activation time. Secondly, under muscle exercise, venous blood draws were only measured at 30-min intervals notably after exercise initiation [4]. After the 30-min interval, uptake under exercise had increased to a maximum. Acetate consumption decreased again to rest level $30 \mathrm{~min}$ after stopping the exercise. In apparent contrast with the observations of acetate turnover in skeletal muscles, heart muscle $\left[{ }^{11} \mathrm{C}\right]$ acetate has been shown to increase rapidly after injection and decrease thereafter to almost background level in between 30 min after injection [5].

We opted for a limited strength isotonic, isometric bilateral abductor exercise of 4.3-min-overall duration. Regarding the short exercise that was thus performed, reliable $\left[{ }^{13} \mathrm{C}\right.$ or $\left.{ }^{14} \mathrm{C}\right]$ acetate physiological observations of skeletal muscle activity were not available. Furthermore, the maximal exercise pressure had to be limited in order not to risk loosening of the freshly implanted hip prothesis.

Bearing in mind the mentioned limitations, the positive outcome of this study appears remarkable. We were able to observe a convincing and reproducible increase in $\left[{ }^{11} \mathrm{C}\right]$ acetate uptake in the major hip abductor muscles after exercise as compared with rest. Although $\left[{ }^{13} \mathrm{C}\right]$ acetate consumption in the active leg had been increased about sixfold as compared with rest [4], the approximate doubling of uptake in $\left[{ }^{11} \mathrm{C}\right]$ acetate PET under exercise compared with rest in our hip arthroplasty patients was highly significant and seems well compatible with an exercise limited in time and force development.

Our data are well compatible with observations of skeletal muscle exercise $\left[{ }^{18} \mathrm{~F}\right] \mathrm{FDG}$ PET [10, 21]. A patchy exercise PET $\left[{ }^{11} \mathrm{C}\right]$ acetate muscle hyperactivity was observed in our patients. This may be due to in-homogenous blood supply which is known to exist under isometric muscle exercise $[22,23]$.

$\left[{ }^{11} \mathrm{C}\right]$ acetate dual muscle PET may thus prove to be an interesting new non-invasive tool to directly demonstrate active muscle function and its occasional impairment after arthroplasty. It may represent an objective method to validate the introduction of new techniques [24, 25] in orthopedic surgery or for studying postsurgical long-term impairment of muscle function and its repair. It may also be an interesting complement to the electro-myogram or MRI [26] and allow an in-depth evaluation of activation failures which could occasionally also be of central nature.

\section{Conclusion}

$\left[{ }^{11} \mathrm{C}\right]$ acetate dual skeletal muscle PET under rest and exercise was successfully performed in a single session on four occasions in two patients. The patients having undergone hip arthroplasty by two different approaches had shown impaired muscle activity to the different muscle groups concerned which are closely related with the surgical procedure. Good recovery of the transiently stunned muscle groups was then observed in both patients at 3 months after completion of their reeducation program. Taken together, these data indicate a potential usefulness of $\left[{ }^{11} \mathrm{C}\right]$ acetate muscle function PET in arthroplasty patients to study different surgical approaches in relation to potential transient or occasional persistently impaired muscle functions. These observations clearly merit further evaluation.

Acknowledgments. We are grateful to Mrs Frances Godson for reviewing the manuscript.

Conflict of Interests. The authors declare that they have no conflict of interest.

Open Access. This article is distributed under the terms of the Creative Commons Attribution Noncommercial License which permits any noncommercial use, distribution, and reproduction in any medium, provided the original author(s) and source are credited.

\section{References}

1. Skutches CL, Holroyde CP, Myers RN, Paul P, Reichard GA (1979) Plasma acetate turnover and oxidation. J Clin Invest 64:708-713

2. Pouteau E, Piloquet H, Maugeais P, Champ M, Dumon H, Nguyen P, Krempf M (1996) Kinetic aspects of acetate metabolism in healthy humans using [1-13C] acetate. Am J Physiol 271:E58-E64

3. Mittendorfer B, Sidossis LS, Walser E, Chinkes DL, Wolfe RR (1998) Regional acetate kinetics and oxidation in human volunteers. Am J Physiol 274:E978-E983

4. van Hall G, Sacchetti M, Radegran G (2002) Whole body and leg acetate kinetics at rest, during exercise and recovery in humans. J Physiol 542:263-272

5. Higashi K, Ueda Y, Matsunari I, Kodama Y, Ikeda R, Miura K, Taki S, Higuchi T, Tonami H, Yamamoto I (2004) 11C-acetate PET imaging of lung cancer: comparison with 18F-FDG PET and 99mTc-MIBI SPET. Eur J Nucl Med Mol Imaging 31:13-21

6. Soloviev D, Tamburella C (2006) Captive solvent [11C]acetate synthesis in GMP conditions. Appl Radiat Isot 64:995-1000

7. Michel C, Sibomana M, Boi A, Bernard X, Lonneux M, Defrise M, Comtat C, Kinahan PE, Townsend DW (1998) Preserving Poisson characteristics of PET data with weighted OSEM reconstruction. IEEE Nuclear Science Symp Medical Imaging Conf 2:1323-1329

8. Kemppainen J, Fujimoto T, Kalliokoski KK, Viljanen T, Nuutila P, Knuuti J (2002) Myocardial and skeletal muscle glucose uptake during exercise in humans. J Physiol 542:403-412

9. Allal AS, Slosman DO, Kebdani T, Allaoua M, Lehmann W, Dulguerov P (2004) Prediction of outcome in head-and-neck cancer patients using the standardized uptake value of 2-[18F]fluoro-2-deoxy-D-glucose. Int J Radiat Oncol Biol Phys 59:1295-1300

10. Pappas GP, Olcott EW, Drace JE (2001) Imaging of skeletal muscle function using (18)FDG PET: force production, activation, and metabolism. J Appl Physiol 90:329-337

11. Schelbert HR (1987) Positron emission tomography. Diagnostic and therapeutic implications in human myocardial ischemia. Int $\mathrm{J}$ Card Imaging 2:199-208

12. Beanlands RS, Bach DS, Raylman R, Armstrong WF, Wilson V, Montieth M, Moore CK, Bates E, Schwaiger M (1993) Acute effects of dobutamine on myocardial oxygen consumption and cardiac efficiency measured using carbon-11 acetate kinetics in patients with dilated cardiomyopathy. J Am Coll Cardiol 22:1389-1398

13. Bengel FM, Ueberfuhr P, Nekolla S, Ziegler SI, Reichart B, Schwaiger $M$ (1999) Oxidative metabolism of the transplanted human heart assessed by positron emission tomography using C-11 acetate. Am J Cardiol 83:1503-1505, A8 
14. Ng CK, Huang SC, Schelbert HR, Buxton DB (1994) Validation of a model for $[1-11 C]$ acetate as a tracer of cardiac oxidative metabolism. Am J Physiol 266:H1304-H1315

15. Armbrecht JJ, Buxton DB, Brunken RC, Phelps ME, Schelbert HR (1989) Regional myocardial oxygen consumption determined noninvasively in humans with [1-11C]acetate and dynamic positron tomography. Circulation 80:863-872

16. Armbrecht JJ, Buxton DB, Schelbert HR (1990) Validation of [1-11C] acetate as a tracer for noninvasive assessment of oxidative metabolism with positron emission tomography in normal, ischemic, postischemic, and hyperemic canine myocardium. Circulation 81:1594-1605

17. Buck A, Wolpers HG, Hutchins GD, Savas V, Mangner TJ, Nguyen N, Schwaiger M (1991) Effect of carbon-11-acetate recirculation on estimates of myocardial oxygen consumption by PET. J Nucl Med 32:1950-1957

18. Wolpers HG, Buck A, Nguyen N, Marcowitz PA, Armstrong WF, Starling MR, Hicks R, Mangner TJ, Schwaiger M (1994) An approach to ventricular efficiency by use of carbon 11-labeled acetate and positron emission tomography. J Nucl Cardiol 1:262-269

19. Sidossis LS, Coggan AR, Gastaldelli A, Wolfe RR (1995) Pathway of free fatty acid oxidation in human subjects. Implications for tracer studies. J Clin Invest 95:278-284

20. Kudo T, Hata T, Kagawa S, Kishibe Y, Iwasaki J, Nakano A, Okazawa H (2008) Simple quantification of myocardial perfusion by pixel-bypixel graphical analysis using carbon-11 acetate: comparison of the K- complexes of carbon-11 acetate and nitrogen-13 ammonia. Nucl Med Commun 29:679-685

21. Ohnuma M, Sugita T, Kokubun S, Yamaguchi K, Rikimaru H (2006) Muscle activity during a dash shown by 18F-fluorodeoxyglucose positron emission tomography. J Orthop Sci 11:42-45

22. Laaksonen MS, Kalliokoski KK, Kyrolainen H, Kemppainen J, Teras M, Sipila H, Nuutila P, Knuuti J (2003) Skeletal muscle blood flow and flow heterogeneity during dynamic and isometric exercise in humans. Am J Physiol Heart Circ Physiol 284:H979-H986

23. Kalliokoski KK, Kuusela TA, Nuutila P, Tolvanen T, Oikonen V, Teras M, Takala TE, Knuuti J (2001) Perfusion heterogeneity in human skeletal muscle: fractal analysis of PET data. Eur J Nucl Med 28:450 456

24. Berry DJ (2005) "Minimally invasive" total hip arthroplasty. J Bone Joint Surg Am 87:699-700

25. Muller M, Tohtz S, Springer I, Dewey M, Perka C (2010) Randomized controlled trial of abductor muscle damage in relation to the surgical approach for primary total hip replacement: minimally invasive anterolateral versus modified direct lateral approach. Arch Orthop Trauma Surg. doi:10.1007/s00402-010-1117-0

26. Pfirrmann CW, Notzli HP, Dora C, Hodler J, Zanetti M (2005) Abductor tendons and muscles assessed at MR imaging after total hip arthroplasty in asymptomatic and symptomatic patients. Radiology 235:969-976 